\title{
Modelling the effect of vertical mixing on bottle incubations for determining in situ phytoplankton dynamics. II. Primary production
}

\author{
Oliver N. Ross ${ }^{1, *}$, Richard J. Geider ${ }^{2}$, Jaume Piera ${ }^{1}$ \\ ${ }^{1}$ Mediterranean Centre for Marine \& Environmental Research, Unidad de Tecnología Marina (UTM, CSIC), \\ Passeig Marítim de la Barceloneta 37-49, 08003 Barcelona, Spain \\ ${ }^{2}$ Dept. Biological Sciences, University of Essex, Wivenhoe Park, Colchester CO4 3SQ, UK
}

\begin{abstract}
The estimation of in situ phytoplankton primary production is pivotal to many questions in biological oceanography and marine ecology both in a local and global context. Applications range from earth system modelling, the characterisation of aquatic ecosystem dynamics, or the local management of water quality. A common approach for estimating in situ primary production is to incubate natural phytoplankton assemblages in clear bottles at a range of fixed depths and to measure the uptake of carbon $\left({ }^{14} \mathrm{C}\right)$ during the incubation period (typically $24 \mathrm{~h}$ ). One of the main concerns with using fixed-depth bottle incubations is whether stranding samples at fixed depths biases the measured $\mathrm{CO}_{2}$ fixation relative to the 'true' in situ mixed conditions. Here we employ an individual based turbulence and photosynthesis model, which also accounts for photoacclimation and -inhibition, to examine whether the in vitro productivity estimates obtained from fixed-depth incubations are representative of the in situ productivity in a freely mixing water column. While previous work suggested that in vitro estimates could either over- or underestimate the in situ productivity, we show that the errors due to arresting the incubation bottles at fixed depths are indeed minimal. We present possible explanations for how previous authors could have arrived at contradictory results and discuss whether they might be artefacts related to the particular sampling protocol used. We discuss the errors associated with chlorophyll-based incubation methods for determining in situ phytoplankton growth rates in Ross et al. (2011; Mar Ecol Prog Ser 435:13-31).
\end{abstract}

KEY WORDS: Bottle incubations - Yo-yo technique - Primary production - Photoacclimation · Photoinhibition $\cdot$ Individual based modelling $\cdot$ Turbulence $\cdot$ Surface mixing layer

\section{INTRODUCTION}

Determining the spatial and temporal (interannual, seasonal, day-to-day) variability of primary productivity remains one of the major goals of biological oceanography (Barber \& Hilting 2002). Phytoplankton productivity underpins pelagic food webs and drives important biogeochemical transformations in the global carbon, nitrogen, phosphorus and sulphur cycles (Falkowski et al. 1998). Coastal and open ocean phytoplankton accounts for about $50 \%$ of global primary productivity (Friend et al. 2009). These calculations are based on remote sensing data, using models that have been derived from and calibrated against ship-based observations. Despite the importance of these ship-based productivity measurements, the standard approach, which involves measurement of the incorporation of ${ }^{14} \mathrm{CO}_{2}$ into organic matter (SteemannNielsen 1952, Strickland \& Parsons 1977, Parsons et al. 1985), is subject to a wide range of methodological variations (Li \& Maestrini 1993). A good case can be made that the measurement of greatest interest is the daily rate of primary production, which is typically determined from dawn-to-dusk or dawn-to-dawn in situ incubations in bottles distributed throughout the vertical light gradient of the euphotic zone (Lohrenz et 
al. 1992). In situ incubations help to ensure that the phytoplankton is exposed to a natural light field (in terms of spectral quality and vertical attenuation) and temperature during the incubation. Despite the ubiquity of its use, some questions still remain about whether ${ }^{14} \mathrm{C}$ assimilation measures net or gross photosynthesis (or something in between) and whether the commonly employed manipulations yield results that are biased (Li \& Maestrini 1993, Furnas 2002).

In a surface mixing layer (SML), vertical mixing by turbulence constantly moves phytoplankton cells through the vertical light gradient (Lewis et al. 1984b), exposing them to a wide range of light intensities (see Fig. 1 in Ross et al. 2008) which may result in considerable stress for the photosynthetic apparatus. Phytoplankton organisms possess a number of mechanisms to cope with exposure to sub- and supra-optimal light levels (MacIntyre et al. 2000). They respond to these changes through the processes of photoacclimation (changes of cellular pigment content) and photoprotection (reducing the efficiency of excitation energy transfer from pigments to reaction centres) (Marra 1978, Lewis et al. 1984a, Geider et al. 1998). Failure to alleviate the stress by excess light may result in photoinhibition of photosynthesis and, in extreme cases, cell death (Eilers \& Peeters 1988, Long et al. 1994, Behrenfeld et al. 1998, Ross et al. 2008). These processes are relevant because the relationship between chlorophyll synthesis, photosynthesis, and cell growth will vary markedly with the cell's light history. Fixed-depth bottle incubations cannot mimic this temporal variability in the cells' individual light histories and it is thus not clear a priori whether cells incubated at a fixed depth (and thus at relatively stable light conditions) will have the same depth-integrated productivity as cells that are moved freely through the SML by turbulence. The magnitude and time course of the light variability depends on the attenuation coefficient, the turbulence intensity and the depth of the surface mixing layer.

With our current knowledge and technology it is not possible to measure routinely the productivity in natural cells that move freely through the water column (but see Hendricks et al. 2005 for some open water methods based on triple isotopes). Alternative approaches have been devised which aim to alleviate this problem by simulating the large-scale displacements due to turbulence using mechanical winches to move the incubation bottles up and down through the water column or using on-deck incubators that expose the samples to random variations in light intensity (Marra 1978, Köhler 1997, Köhler et al. 2001, Gocke \& Lenz 2004). While both the yo-yo-technique and the linear incubator appear to be promising approaches to overcome part of the limitations associated with fixeddepth incubations, they both come with the caveat that the choice of the amplitude and period of the vertical oscillations/light variations is an ad hoc decision which is often taken without much prior knowledge of the in situ mixing conditions.

Previous work investigated the problems associated with fixed-depth measurements of primary productivity. Barkmann \& Woods (1996) employed a similar individual based approach to model the physical trajectories of Lagrangian ensembles of cells. Using a biological model that was not cell-based, and a treatment of photoacclimation that was more empirical and specific to Thalassiosira pseudonana, they found that fixed-depth bottle incubations overestimated primary productivity (carbon production) by up to $40 \%$. Their results stand in contrast to the findings by Marra (1978) who concluded, based on a comparison between oscillating and fixed-depth bottle incubations, that the fixed-depth incubation could underestimate the depthintegrated in situ productivity by up to $87 \%$. Gallegos \& Platt (1982) used a linear incubator to test the effect of vertical mixing on bottle incubations and found similar results to Marra (1978). However, in both Marra (1978) and Gallegos \& Platt (1982) the largest underestimate in the fixed-bottle incubations relative to the 'yo-yo' bottle incubations occurred when the sample was taken from one depth deep in the euphotic zone. In an experiment in a tidally energetic and thus highly turbulent coastal area, with phytoplankton cells that showed no signs of photoinhibition, Yoder \& Bishop (1985) found no significant differences in production between fixed-depth incubations and bottles that were oscillated at various rates through the water column. Using dimensional arguments, Gallegos \& Platt (1985) concluded that fixed-depth incubations could significantly (by 100\%) underestimate the depth-integrated in situ production if the cells show signs of photoinhibition. Mallin \& Paerl (1992) found that in turbid water columns static in vitro incubations would underestimate the in situ productivity by up to $15 \%$, which they attributed to the alleviating effect of mixing on photoinhibition.

In the present study, we employ a state-of-the-art individual-based model of turbulence and phytoplankton growth, acclimation, and inhibition to compare the primary production in fixed-depth bottle incubations with the production of cells that are freely mixing in the SML (see Ross et al. 2011, this volume, for details).

\section{METHOD}

The model used here is identical to the one described in Ross et al. (2011).

As we examine issues related to the light history and thus the vertical light variability, our problem can be 
reduced to one dimension. It is thus sufficient to employ a 1D vertical physical-biological model. The model consists of 2 main compartments: (1) a Lagrangian particle tracking model to describe the vertical displacement of cells in response to turbulence (Ross \& Sharples 2004), and (2) a biological component which calculates the primary production and growth rate based on the individual light histories, taking into account the effects of photoacclimation and photoinhibition. Table 1 contains a summary of the main model parameters and their standard values.

The biological model used for the comparison is based on the RGI model in Ross \& Geider (2009) and the full set of equations and associated parameters is contained in Ross et al. (2011; their Appendix 1). To quantify the errors we focus on how primary production is affected by the fixed-depth incubation procedure. For each depth bin, we therefore calculate the carbon production per unit volume during the fixeddepth incubations, $P_{\text {incub }}(\mathrm{z})$, and compare it with the production in the freely mixing $\mathrm{SML}, P_{\text {mixed }}(\mathrm{z})$. The difference is displayed as the percent over- or underestimation

$$
\Pi(z)=\frac{P_{\text {incub }}(z)-P_{\text {mixed }}(z)}{\left|P_{\text {mixed }}(z)\right|} \cdot 100 \%
$$

or depth integrated as

$$
\bar{\Pi}=\frac{\sum_{z=0}^{-H} P_{\text {incub }}(z)-\sum_{z=0}^{-H} P_{\text {mixed }}(z)}{\left|\sum_{z=0}^{-H} P_{\text {mixed }}(z)\right|} \cdot 100 \%
$$

Table 1. List of commonly used symbols thoughout the text. A list of the remaining model parameters for the biological model can be found in Table 2 in Ross et al. (2011) and Table 1 in Ross \& Geider (2009). Data based on experiments with

\begin{tabular}{|c|c|c|c|}
\hline Symbol & Description & Value & Unit \\
\hline$a^{C h l}$ & Chl a specific absorption coefficient & 6.56 & $\mathrm{~m}^{2} \mathrm{gChl}^{-1}$ \\
\hline$C^{F}$ & Amount of carbon in functional pool & Variable & $\mathrm{gC} \mathrm{cell}^{-1}$ \\
\hline$C$ & Total amount of carbon in cell & Variable & gC cell ${ }^{-1}$ \\
\hline $\mathrm{Chl}$ & Amount of chlorophyll $a$ in cell & Variable & $\mathrm{gChl} \mathrm{cell}^{-1}$ \\
\hline$D$ & Depth of water column (model domain) & Variable & $\mathrm{m}$ \\
\hline$H$ & Surface mixed layer (SML) thickness & Variable & $\mathrm{m}$ \\
\hline$I$ & Irradiance & Variable & $\mu \mathrm{mol}$ photons $\mathrm{m}^{-2} \mathrm{~s}^{-1}$ \\
\hline$I_{m}$ & Maximum noon irradiance & Variable & $\mu \mathrm{mol}$ photons $\mathrm{m}^{-2} \mathrm{~s}^{-1}$ \\
\hline$k$ & PAR attenuation coefficient & Variable & $\mathrm{m}^{-1}$ \\
\hline$k_{r}$ & Repair rate from photoinhibition & $4.5 \times 10^{-5}$ & $\mathrm{~s}^{-1}$ \\
\hline$k_{d}$ & Damage probability for photoinhibition & $1.4 \times 10^{-7}$ & - \\
\hline$k$ & Turbulent diffusivity & Variable & $\mathrm{m}^{2} \mathrm{~s}^{-1}$ \\
\hline$K_{m}$ & Mid SML turbulent diffusivity & Variable & $\mathrm{m}^{2} \mathrm{~s}^{-1}$ \\
\hline$P^{\text {cell }}$ & Cell-based C production & Variable & $\mathrm{gC}$ cell $^{-1} \mathrm{~s}^{-1}$ \\
\hline$P_{\max }^{\text {cell }}$ & Max. cell-based C production & Variable & gC cell ${ }^{-1} \mathrm{~s}^{-1}$ \\
\hline$P_{m}^{c h l}$ & Max. Chl-based C production (with inhibition) & $P_{\max }^{c h l} \vartheta$ & $\mathrm{gC} \mathrm{gChl}^{-1} \mathrm{~s}^{-1}$ \\
\hline$P_{\max }^{c h l}$ & Max. Chl-based C production (without inhibition) & $3.3 \times 10^{-3}$ & $\mathrm{gC} \mathrm{gChl}^{-1} \mathrm{~s}^{-1}$ \\
\hline$P_{\max }^{F}$ & Max. $\mathrm{C}^{\mathrm{F}}$-based production at a given $\mathrm{N}: \mathrm{C}$ & Variable & $\mathrm{s}^{-1}$ \\
\hline$z$ & Particle position & $<0$ & $\mathrm{~m}$ \\
\hline$\alpha_{\max }^{C h l}$ & Initial slope of the P-I curve (without inhibition) & $6.3 \times 10^{-6}$ & $\mathrm{gC} \mathrm{m}^{2}(\mathrm{gChl} \mu \mathrm{mol} \text { photons })^{-1}$ \\
\hline$\alpha^{C h l}$ & Initial slope of the P-I curve (with inhibition) & $\alpha_{\max }^{C h l} \cdot \vartheta$ & $\mathrm{gC} \mathrm{m}^{2}(\mathrm{gChl} \mu \mathrm{mol} \text { photons })^{-1}$ \\
\hline$\Delta t$ & Model time step & $1-6^{\mathrm{a}}$ & $\mathrm{s}$ \\
\hline$\phi_{m}$ & Achieved quantum yield when inhibited & Variable & $\mathrm{gC}(\mu \mathrm{mol} \text { photons })^{-1}$ \\
\hline$\phi_{\max }$ & Max. quantum yield of photosynthesis & $0.96 \times 10^{-6}$ & $\mathrm{gC}(\mu \mathrm{mol} \text { photons })^{-1}$ \\
\hline$P_{\text {incub }}$ & Depth dependent productivity from incubations & Variable & $\mathrm{gC} \mathrm{m}^{-3} \mathrm{~h}^{-1}$ \\
\hline$P_{\text {mixed }}$ & Depth dependent productivity in turbulent water & Variable & $\mathrm{gC} \mathrm{m}^{-3} \mathrm{~h}^{-1}$ \\
\hline$\Pi$ & Error between $P_{\text {incub }}$ and $P_{\text {mixed }}$ & Variable & $\%$ \\
\hline $\bar{\Pi}$ & Error between depth integrated $P_{\text {incub }}$ and $P_{\text {mixed }}$ & Variable & $\%$ \\
\hline$\theta^{C h l}$ & Chl:C ratio & Variable & $\mathrm{gChl}(\mathrm{gC})^{-1}$ \\
\hline$\vartheta$ & Proportion of functional PSII reaction centres & $0 \leq \vartheta \leq 1$ & - \\
\hline$\sigma_{\mathrm{PSII}}$ & Absorption cross-section of PSII & 1.5 & $\mathrm{~m}^{2}\left(\mu \mathrm{mol}\right.$ photons $\left.{ }^{-1}\right)$ \\
\hline$\xi$ & Optical depth within water column & Variable & - \\
\hline$\xi_{\mathrm{SML}}$ & Optical depth of the surface mixing layer & $H \cdot k$ & - \\
\hline$\tau_{m}$ & Mixing time scale of surface mixing layer & $H^{2} / K_{m}$ & $\mathrm{~s}$ \\
\hline
\end{tabular}
Skeletonema costatum 
$P_{\text {incub }}(z)$ represents the production per unit volume of all cells present within a certain depth bin during the $24 \mathrm{~h}$ incubation period on the last day of those model runs where the turbulence had been switched off. $P_{\text {mixed }}(z)$ is the reference production from those experiments where the turbulence had been kept switched on during the 5th day of the simulations and is thus the production per unit volume of all cells passing through a particular depth bin during the $24 \mathrm{~h}$ incubation period. Both production values have units $\left(\mathrm{gC} \mathrm{m}^{-3} \mathrm{~d}^{-1}\right)$. As we are not interested in absolute magnitudes but only in differences between the mixed and fixed-depth scenarios, we display those units as 'arbitrary' in the plots. As most experimentalists use the ${ }^{14} \mathrm{C}$ incubation method at a range of depths throughout the SML, we focus mainly on the error in the depth-integrated production $\bar{\Pi}$.

\section{RESULTS}

Fig. 1 shows a comparison of the 2 productivities for a range of environmental conditions. In agreement with our findings regarding the growth rates (Ross et al. 2011), the error in the productivity scales with both $\tau_{m}$ and $\xi_{\text {SML }}$ (Fig. 1a-d), in the sense that the vertical distribution of $\Pi$ and the depth-integrated error $\bar{\Pi}$ do not depend on factors such as SML depth, turbulence intensity, or light attenuation per se, but rather on the derived parameters $\tau_{m}$ and $\xi_{\text {SML }}$. The results in Fig. 1a,b were obtained for a shallow $(H=6 \mathrm{~m})$ and relatively turbid $\left(k=0.9 \mathrm{~m}^{-1}\right)$ SML which might be representative of an estuary or coastal lagoon. The optical depth of this SML is $\xi_{\mathrm{SML}}=5.4$ and the mixing time scale $\tau_{m}=$ $100 \mathrm{~h}$. The same trends and magnitudes in the associated error are obtained for an open ocean setting $(H=$ $60 \mathrm{~m}$ and $k=0.09 \mathrm{~m}^{-1}$ ), as long as both $\xi_{\mathrm{SML}}$ and $\tau_{\mathrm{m}}$ are (a) Productivity

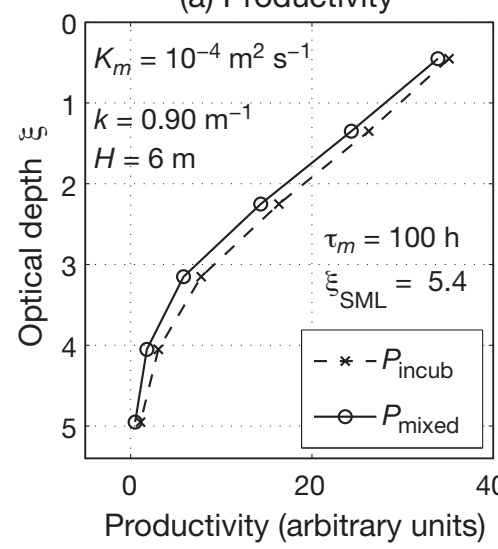

(e) Productivity

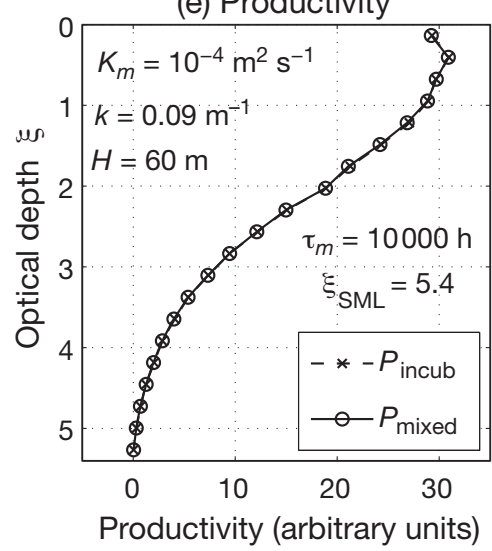

(b) Error П

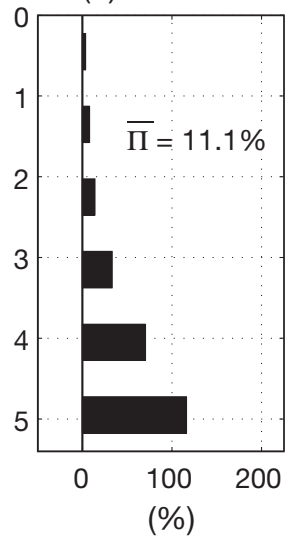

(f) Error $\Pi$

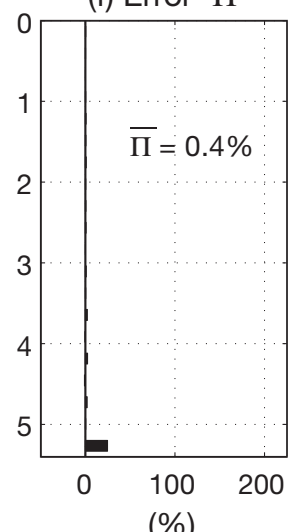

(c) Productivity

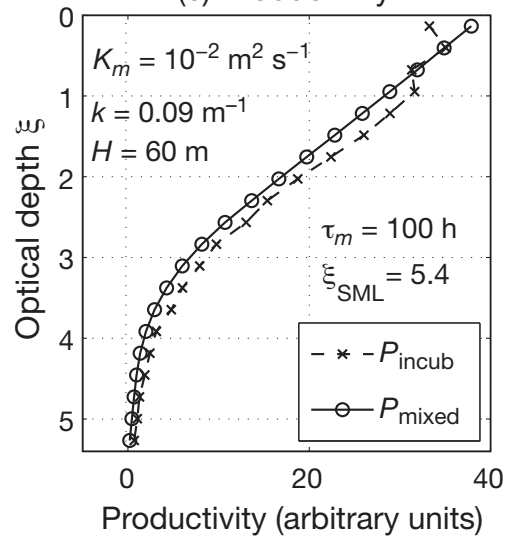

(g) Productivity

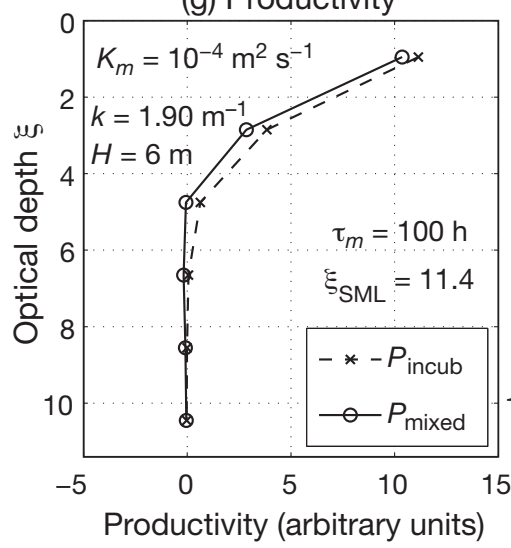

(d) Error $\Pi$

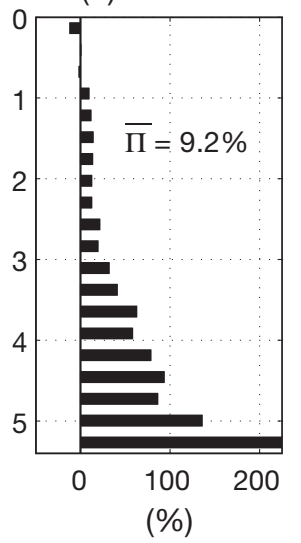

(h) Error П

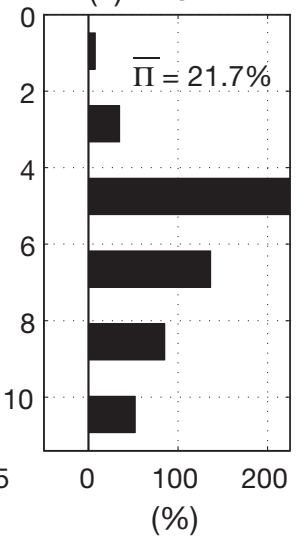

Fig. 1. Comparing the productivities and associated errors for 4 different scenarios. The 2 sets of results, $(\mathrm{a}, \mathrm{b})$ and $(\mathrm{c}, \mathrm{d})$, are for a shallow and deep surface mising layer (SML) respectively and the parameters are chosen such that the optical depth $\xi_{\mathrm{SML}}=5.4$ and the mixing time scale $\tau_{m}=100 \mathrm{~h}$ are the same for both set-ups. In scenario $(\mathrm{e}, \mathrm{f})$ the mixing time scale is larger by a factor 100 , while in $(g, h)$ the optical depth of the SML, $\xi_{\mathrm{SML}}$, has been increased. The vertical resolution for model output was always $1 \mathrm{~m}$, but for better readability we used vertical bin sizes of $3 \mathrm{~m}$ for the graphical output in the deep $(H=60 \mathrm{~m})$ scenarios. See 
unchanged (Fig. 1c,d). Compared to Fig. 1a, we observe a depression in $P_{\text {incub }}$ at the surface in Fig. 1c. In the shallow but turbid water column of Fig. 1a, the particles in the top $1 \mathrm{~m}$ bin receive a lower light dose compared to the particles in the top $1 \mathrm{~m}$ bin of the deep but less turbid water column of Fig. 1c. As a result, the latter cells show more pronounced signs of photoinhibition, which leads to the observed depression in $P_{\text {incub }}$ and a slightly lower $\bar{\Pi}$. The total productivity in the 10 times deeper SML is of course 10 times higher as in the shallow water column, mainly because we have 10 times as many model particles in the deep SML (80 000 in the deep vs 8000 in the shallow). The consistency between both scenarios in terms of the governing parameters $\xi_{\mathrm{SML}}$ and $\tau_{m}$ is further illustrated by the consistency in the Chl:C ratios (Fig. 5 in Ross et al. 2011), which shows the same vertical heterogeneity within and between depth bins for the same values of $\xi_{\mathrm{SML}}$ and $\tau_{m}$. In this experiment, the systematic error due to keeping the bottles at fixed depths is about $10 \%$, which is of the same order of magnitude as the typical uncertainty in calculating productivity (Pemberton et al. 2006). If we decrease the turbulence intensity (increase $\tau_{m}$ ), $\bar{\Pi}$ almost disappears (Fig. 1e,f). Larger depth-integrated errors are possible, however, if we have a more turbid water column (Fig. 1g,h). We never observed $\bar{\Pi}>25 \%$ for the range of environmental conditions we tested (cf. Fig. 2). The total variation of $\bar{\Pi}$ throughout the parameter space is relatively small with a maximum of $25 \%$ and a minimum of $2 \%$.

In general, we found that $\bar{\Pi}$ is positive, which means that the productivity in the fixed-depth incubations is typically higher than the in situ value. Locally, $\Pi(z)$ can become negative near the very surface if photoinhibition leads to a reduction in $P_{\text {incub }}(z)$. While the absolute

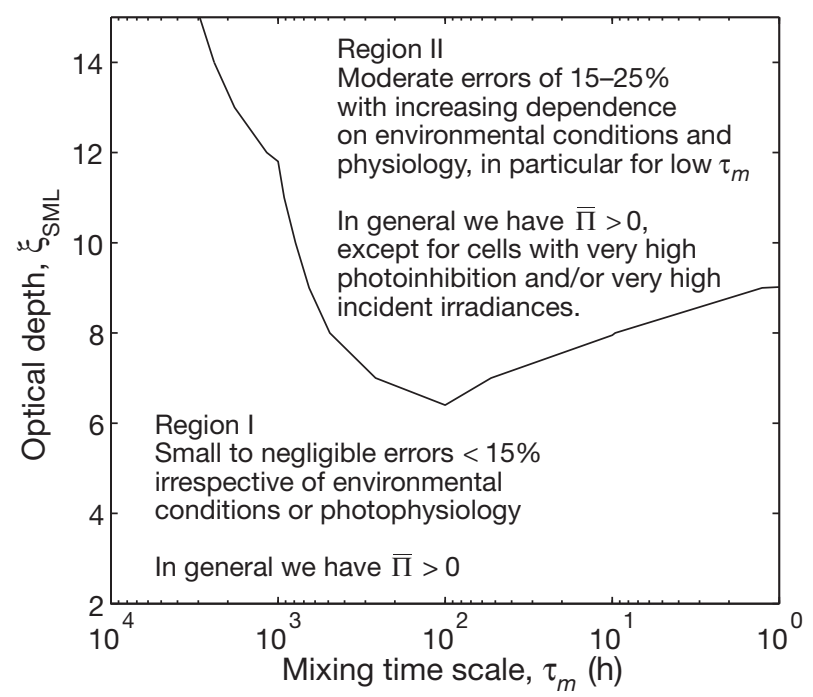

Fig. 2. Summary of the general trends observed in the numerical experiments error generally decreases with depth, the relative error increases because the productivity $P_{\text {mixed }}(z)$ itself decreases and approaches zero. When the optical depth of the SML is large (Fig. $1 \mathrm{~g}, \mathrm{~h}$ ) we obtain a peak in $\Pi$ in the depth bin containing the $1 \%$ light level. $\Pi$ then decreases for greater depths as the productivity in the fixed-bottle incubations (and thus the absolute error) also decreases.

\section{Sensitivity to parameter values}

For most scenarios, the depth-integrated error is small $(<15 \%)$ and remains small even if we change the environmental conditions or the physiological parameters. Only for high values of $\xi_{\mathrm{SML}}$ in combination with low values of $\tau_{m}$, did we observe a sensitivity to changes in some parameter values. This is illustrated in Fig. 3a,b which shows the results for a scenario from Region II in Fig. 2 where we observed relatively large errors. Due to the high eddy diffusivity $\left(\tau_{m}=1 \mathrm{~h}\right)$, we have $P_{\text {incub }}<$ $P_{\text {mixed }}$ at the surface because the level of photoinhibition is higher in the stationary bottles (Fig. 4a). The euphotic depth is at about $\xi \approx 4.4$ where $P_{\text {mixed }}$ turns negative (the $1 \%$ light depth is at $\xi=4.6$ ). The reason why $P_{\text {mixed }}$ turns negative while $P_{\text {incub }}$ remains at, or slightly above, zero is that in the mixed case, turbulence brings cells with a relatively high carbon content from the well lit euphotic zone near the surface to these greater depths, where respiration exceeds photosynthesis, leading to a rapid reduction of reserve carbon pools and hence to $P_{\text {mixed }}<$ 0 . In the fixed-depth incubations, the cells in the lower SML have already depleted their carbon reserves (having come through the night) and a further reduction is therefore not possible, as they are at the subsistence threshold.

If we triple the probability of photodamage (model parameter $k_{d}$ ) and increase the maximum noon irradiance, $I_{m}$, from 650 to $850 \mathrm{~W} \mathrm{~m}^{-2}$, the productivity in the stationary bottles is strongly affected (Fig. 3c vs a). While $P_{\text {mixed }}$ is only slightly lower near the surface, $P_{\text {in- }}$ cub is significantly reduced and $\Pi$ almost reaches $-70 \%$ in the surface bin (Fig. 3d). As a result, the error for the depth-integrated productivity $\bar{\Pi}$ has also become negative. This is caused by the increased levels of photoinhibition reached in the fixed-depth bottles (Fig. 4b) where $\vartheta$ reaches as low as 0.17 near the surface compared to 0.45 with the 'standard' inhibition and irradiance (Fig. 4a).

A reduction in the initial slope of the P-I curve, $\alpha_{\max }^{C h l}$ leads to a reduction in $\bar{\Pi}$ (Fig. 3e,f) and also affects the level of photoinhibition (Fig. 3c), because the photodamage probability, $k_{d}$, depends on the rate of photon absorption (Eq. 3). In addition, a lower value for $\alpha_{\max }^{C h l}$ produced a different $\mathrm{Chl}: \mathrm{C}$ distribution: by reducing 
(a) Standard biology

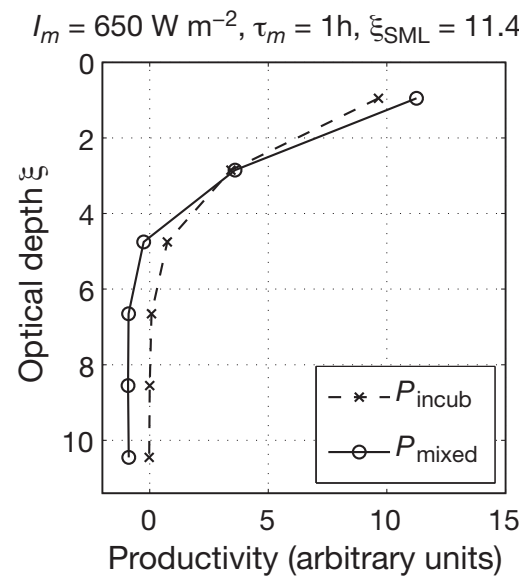

(e) $1 / 3 \alpha^{C h l}$

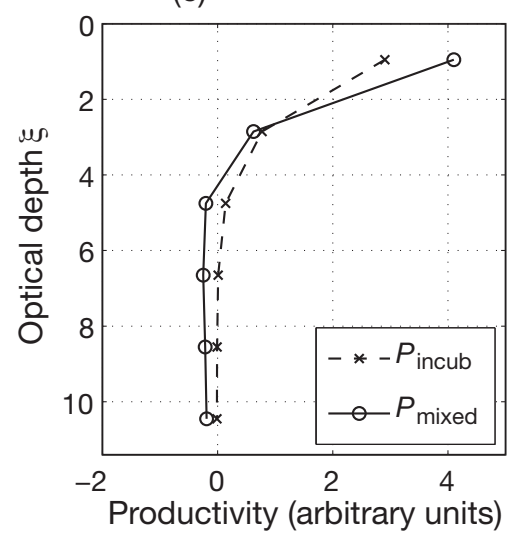

(b) Error П

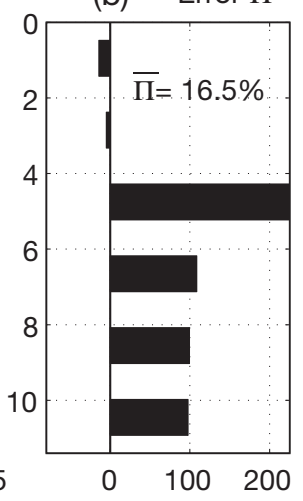

(\%) (f) Error $\Pi$

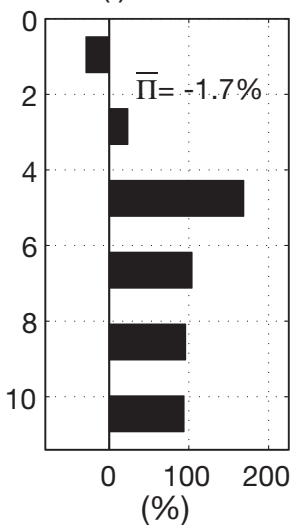

(c) triple $k_{d}, I_{m}=850 \mathrm{~W} \mathrm{~m}^{-2}$

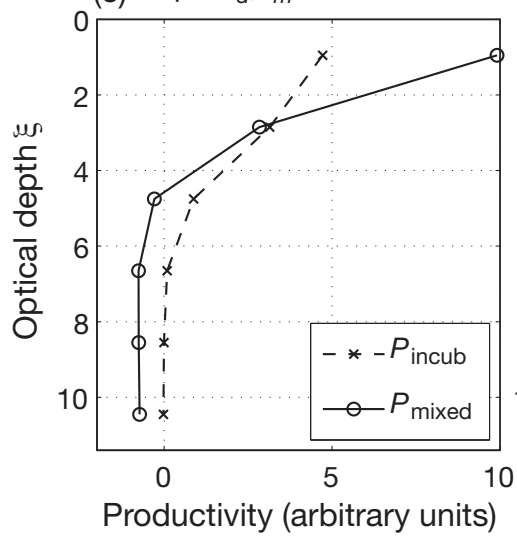

(g) triple $k_{d}, I_{m}=850 \mathrm{~W} \mathrm{~m}^{-2}$

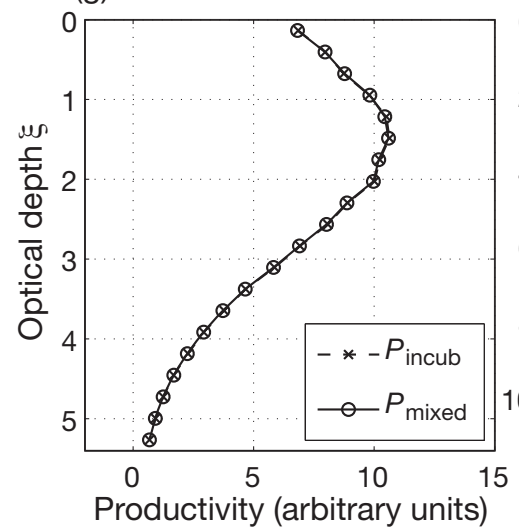

(d) Error П

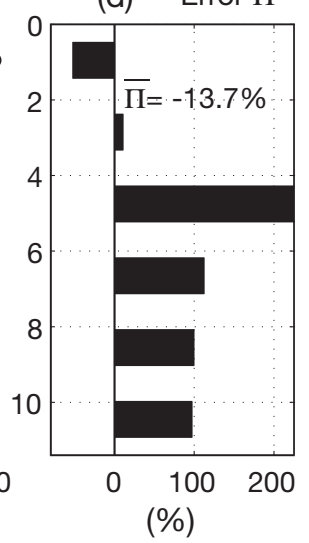

(h) Error ח

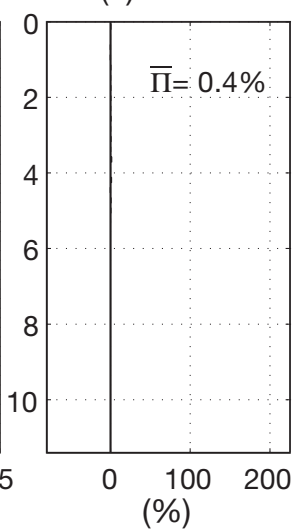

Fig. 3. (a,b) As in Fig. 1g but with a 100 times higher turbulence intensity (100 times lower $\left.\tau_{m}\right)$. (c,d) Compared to scenario (a) we have increased the damage probability $k_{d}$ by a factor of 3 and the midday noon irradiance from 650 to $850 \mathrm{~W} \mathrm{~m}^{-2}$. (e,f) Factor 3 decrease in $\alpha_{\max }^{C h l}$ compared to the scenario in $(a) .(g, h)$ Effect of a factor of 3 higher damage probability in combination with an increase in the midday noon irradiance from 650 to $850 \mathrm{~W} \mathrm{~m}^{-2}$ for the scenario in Fig. 1e

$\alpha_{\max }^{C h l}$ in such a turbid water column, we have reduced the cells' capability to take up carbon, hence the Chl:C ratio of most cells is at their maximum (not shown). Even during the fixed-depth incubations, the surface bottle does not reach the same low values in $\mathrm{Chl}$ as our reference experiment from Fig. 3a. As a result, the relative difference in productivity at the surface changes and $\bar{\Pi}$ decreases.

Scenarios from Region I in Fig. 2 did not exhibit any noticeable sensitivity to changes in the parameter values. If we use the scenario from Fig. 1e and again triple the probability of photoinhibition $\left(k_{d}\right)$ while increasing the maximum noon irradiance to $850 \mathrm{~W} \mathrm{~m}^{-2}$, we find that the productivity maximum shifts away from the surface to greater depths, but the overall error is unaffected (Fig. 3g,h). Due to the high mixing time scale, the distributions of Chl:C (not shown) and also the vertical structure of $\vartheta$ (Fig. $4 \mathrm{~d}$ ) do not exhibit any significant differences between the mixed and stationary scenario.

\section{Sensitivity to incubation time}

The results shown in Fig. 1 \& Table 2 were obtained for an incubation time of $24 \mathrm{~h}$ extending from midnight until midnight on the last day of the simulations. Although the depth-integrated errors are relatively small, they can be further reduced by shortening the incubation time. If the bottles are incubated for only $4 \mathrm{~h}$ from 10:00 to 14:00 h, for instance (cf. Marra 1978), the depth-integrated error is approximately halved (Fig. 5).

\section{Dependence on the mixing time scale}

In general, we found that the in vitro production was higher than in the in situ value. The productivity only shows small variations with changing turbulence intensity (Table 3), but only in the presence of convective overturning at night. The values in Table 3 for $\tau_{m}>$ 
(a) $\tau_{m}=1 \mathrm{~h}$, standard biology
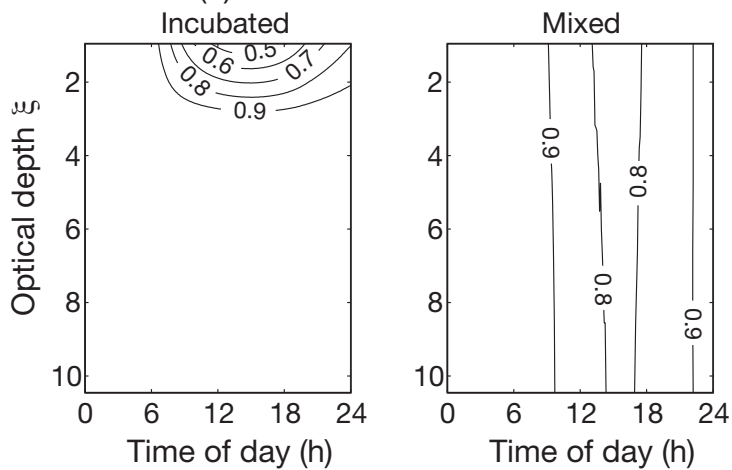

(c) $\tau_{m}=1 \mathrm{~h}, 1 / 3 \alpha^{C h l}$
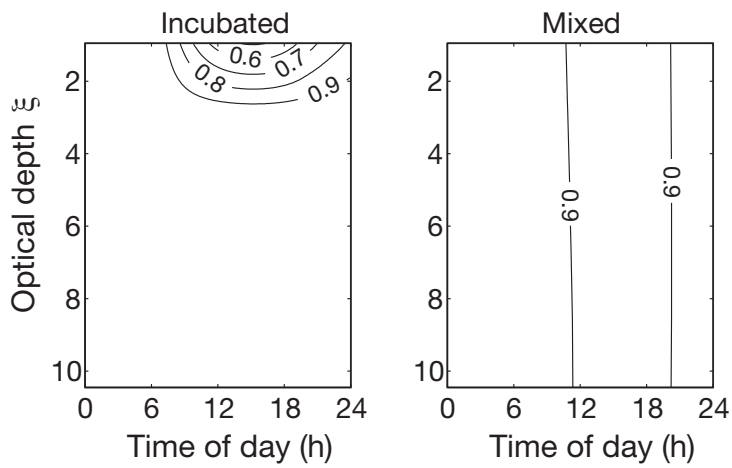

(b) $\tau_{m}=1 \mathrm{~h}$, triple $k_{d}, \mathrm{I}_{m}=850 \mathrm{~W} \mathrm{~m}^{-2}$
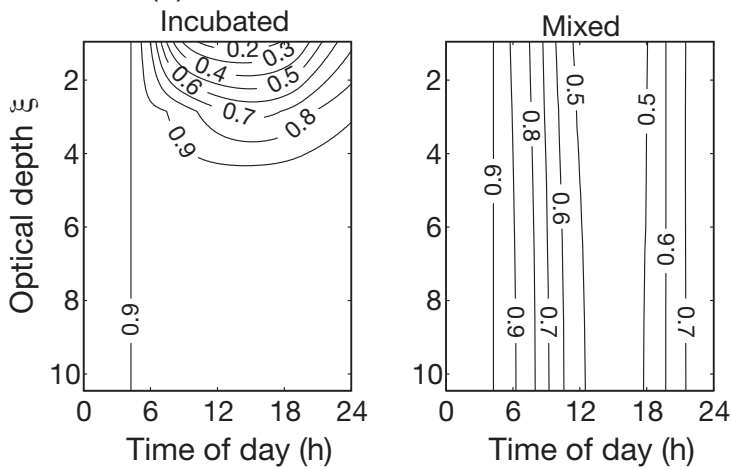

(d) $\tau_{m}=10000 \mathrm{~h}$, triple $k_{d}, \mathrm{I}_{m}=850 \mathrm{~W} \mathrm{~m}^{-2}$ Incubated

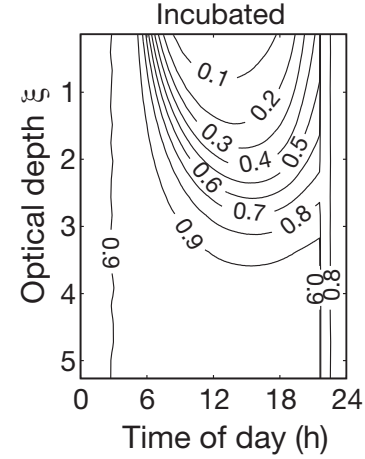

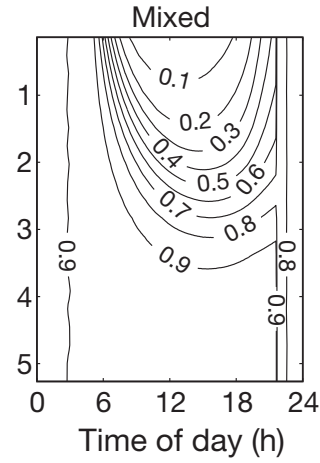

Fig. 4. Photoinhibition parameter $\vartheta$ for the scenarios from Fig. 3. The left panels in each subplot show the amount of inhibition in the stationary incubation bottles while the right panels are for the freely mixing water column. $\vartheta$ describes the proportion of still functional PSII reaction centres $(0 \leq \vartheta \leq 1)$ with the extremes of $\vartheta=1$ corresponding to zero inhibition (i.e. all centres are functional) and the theoretical value of $\vartheta=0$ corresponding to $100 \%$ inhibition (with no functional centres remaining)

$100 \mathrm{~h}$ were obtained from scenarios where the mixing time scale was so high that we considered it to be unrealistic that such a stable SML could be maintained for $5 \mathrm{~d}$ without restratifying and shallowing. In order to avoid experimental artefacts, we therefore applied a

Table 2. Results for the depth-averaged errors for the scenarios shown in Figs. $1 \& 3 . \tau_{m}$ is given in $\mathrm{h}$; units for the remaining environmental parameters can be found in Table 1

\begin{tabular}{|c|c|c|c|c|c|c|}
\hline & \multicolumn{5}{|c|}{ Environmental parameters } & \multirow{2}{*}{$\begin{array}{c}\text { Error } \\
\bar{\Pi}\end{array}$} \\
\hline & $H$ & $K_{m}$ & $k$ & $\tau_{m}$ & $\xi_{\mathrm{SML}}$ & \\
\hline \multicolumn{7}{|c|}{ Fig. 1} \\
\hline $\mathrm{a}$ & 6 & $10^{-4}$ & 0.9 & 100 & 5.4 & 11.1 \\
\hline $\mathrm{c}$ & 60 & $10^{-2}$ & 0.09 & 1 & 5.4 & 9.2 \\
\hline e & 60 & $10^{-4}$ & 0.09 & $10^{4}$ & 5.4 & 0.4 \\
\hline g & 6 & $10^{-4}$ & 1.9 & 100 & 11.4 & 21.7 \\
\hline \multicolumn{7}{|c|}{ Fig. 3} \\
\hline $\mathrm{a}$ & 6 & $10^{-2}$ & 1.9 & 1 & 11.4 & 16.5 \\
\hline \multicolumn{7}{|c|}{ Using triple $k_{d}$ and $I_{m}=850 \mathrm{~W} \mathrm{~m}^{-2}$} \\
\hline $\mathrm{C}$ & 6 & $10^{-2}$ & 1.9 & 1 & 11.4 & -13.7 \\
\hline g & 60 & $10^{-4}$ & 0.09 & $10^{4}$ & 5.4 & 0.4 \\
\hline \multicolumn{7}{|c|}{ Using one third of $\alpha_{\max }^{C h l}$} \\
\hline $\mathrm{e}$ & 6 & $10^{-2}$ & 1.9 & 1 & 11.4 & -1.7 \\
\hline
\end{tabular}

higher diffusivity value at night to simulate convective overturning due to density instabilities. In the absence of such nocturnal mixing, we would have obtained much higher productivity values for $\tau_{m}>100 \mathrm{~h}$, as the lack of vertical mixing would allow for a buildup of biomass near the surface and thus higher production values on Day 5 of the simulations.

\section{DISCUSSION}

The ability to reliably determine the in situ productivity of phytoplankton is important to furthering our understanding of aquatic ecosystems, for accurate climate predictions, and for the management of water quality. Using a modelling approach we found that the depth-integrated productivity from fixed-depth incubations tends to overestimate the net depth-integrated primary productivity (carbon fixation) in the freely mixing water column by up to $25 \%$, but in the majority of cases by less than $15 \%$. This overestimation can be turned into an underestimation of the same order of magnitude if the cells become easily inhibited, i.e. if they have a high probability of photodamage $\left(k_{d}\right)$ 
(a) Productivity

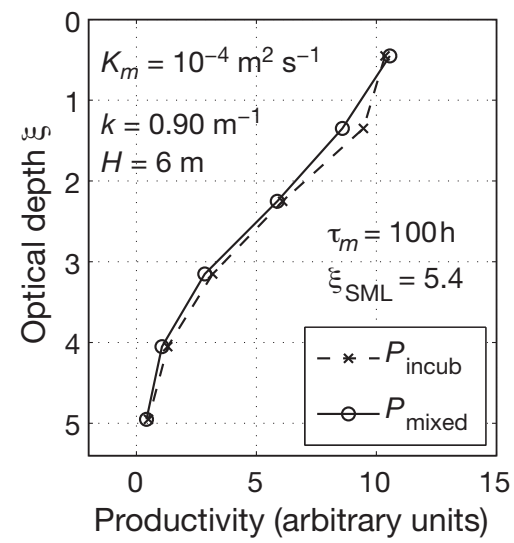

(b) Error $\Pi$

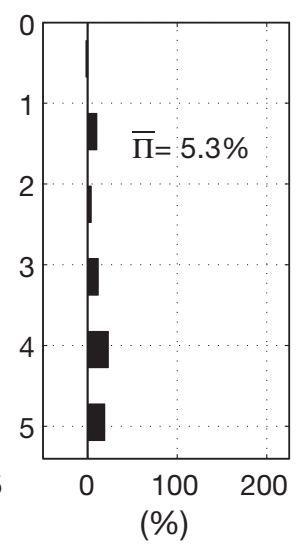

(c) Productivity

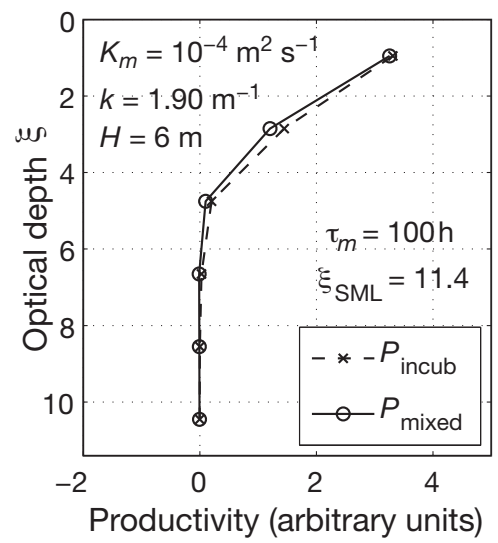

(d) Error ח

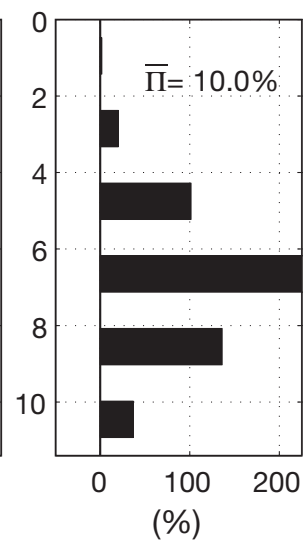

Fig. 5. Corresponding results to panels $(\mathrm{a}, \mathrm{b})$ and $(\mathrm{g}, \mathrm{h})$ in Fig. 1 except that the incubation time was only $4 \mathrm{~h}$ from $10: 00$ to $14: 00 \mathrm{~h}$

Table 3. Normalised productivities on Day 5 of the experiment for a range of turbulence intensities using $H=6 \mathrm{~m}$ and $k=$ $0.9 \mathrm{~m}^{-1}$. The results are normalised to $\tau_{m}=1 \mathrm{~h}$ (freely mixed)

\begin{tabular}{|c|c|c|}
\hline$\tau_{m}(\mathrm{~h})$ & Freely mixed & Incubated \\
\hline 1 & 100 & 111.1 \\
\hline 10 & 95.4 & 105.1 \\
\hline 100 & 106.1 & 117.9 \\
\hline 1000 & $106.7^{\mathrm{a}}$ & $107.8^{\mathrm{a}}$ \\
\hline 10000 & $108.3^{\mathrm{a}}$ & $108.2^{\mathrm{a}}$ \\
\hline \multicolumn{3}{|c|}{$\begin{array}{l}\text { a Mixing time scale was } \tau_{m}>100 \mathrm{~h} \text { and the water column } \\
\text { was mixed rapidly at night using } K_{m}=10^{-2} \mathrm{~m}^{2} \mathrm{~s}^{-1} \text { for } 5 \mathrm{~h} \\
\text { to simulate convective overturning }\end{array}$} \\
\hline
\end{tabular}

(Fig. 3c). This underestimation was also observed in a turbid estuary by Mallin \& Paerl (1992) who found that high photoinhibition in static incubations could lead to an underestimation in productivity of up to $15 \%$.

Compared with other errors these magnitudes are relatively small. Other source of error include those associated with patchy distributions of phytoplankton (Harris \& Smith 1977, Tokarev et al. 1998) (affecting the initial biomass in the bottles), other incubation artifacts (e.g. removal of UV radiation by glass bottles, exclusion of copepods which can impact phytoplankton mortality and nutrient regeneration, inadvertent introduction of toxic contaminants), and uncertainty about just exactly what ${ }^{14} \mathrm{C}$ uptake measures (net vs. gross photosynthesis, cf. Richardson et al. 1984). We did not evaluate whether the ${ }^{14} \mathrm{C}$ incubation technique provides correct values of net primary productivity under the imposed incubation conditions (e.g. Moigis 2000) but only whether productivity estimates from fixed-depth incubation bottles provide a good representation of mixed conditions. We also did not concern ourselves with other sources of error such as bottle effects, which have already been addressed elsewhere (see Furnas 2002).

\section{Mixed vs. mixing layer}

In order to explore a wide range of habitats, we also examined 'mixing' layers which may be physically unrealistic. For instance, a $60 \mathrm{~m}$ SML that is only mixed at $K_{m}=10^{-4} \mathrm{~m}^{2} \mathrm{~s}^{-1}$ has a high mixing time scale of more than $1 \mathrm{yr}$ and is likely to re-stratify which would automatically lead to a shoaling of the SML and thus a lowering of the mixing time scale. The choice to expand our parameter space to such regions was based on data reported in the literature. In Table 1 of Gallegos \& Platt (1985), for instance, the daytime diffusivity value at a station in the oligotrophic ocean was reported as $10^{-5} \mathrm{~m}^{2} \mathrm{~s}^{-1}$. While nothing is said about the depth of the $\mathrm{SML}$, even for a shallow layer of only $10 \mathrm{~m}$ we obtain a mixing time scale of $2500 \mathrm{~h}$, which is one quarter of the maximum used in our experiments (Fig. 2). Such a low daytime turbulence value can only be observed if the SML is mixed more rapidly at night by convective overturning, induced by instabilities due to surface cooling. To increase the realism of our simulations, we therefore incorporated this into our model by temporarily increasing the eddy diffusivity at night for all scenarios with $\tau_{m}>100 \mathrm{~h}$. By invoking convective overturning at night, particles always start the next day randomly distributed through the SML, i.e. nocturnal mixing can bring cells from near the surface, which are high in carbon, to greater depths, where they will reduce their carbon due to maintenance respiration. This downward flux of carbon to the less productive parts of the water column, where it is used for maintenance respiration and therefore no longer available for 
(a) $\mathrm{Chl}: \mathrm{C}$

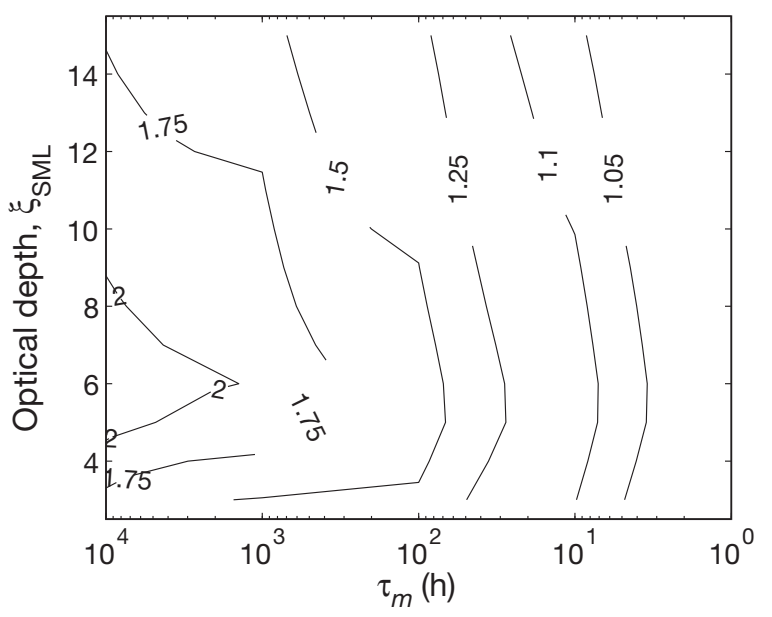

(b) $\vartheta$

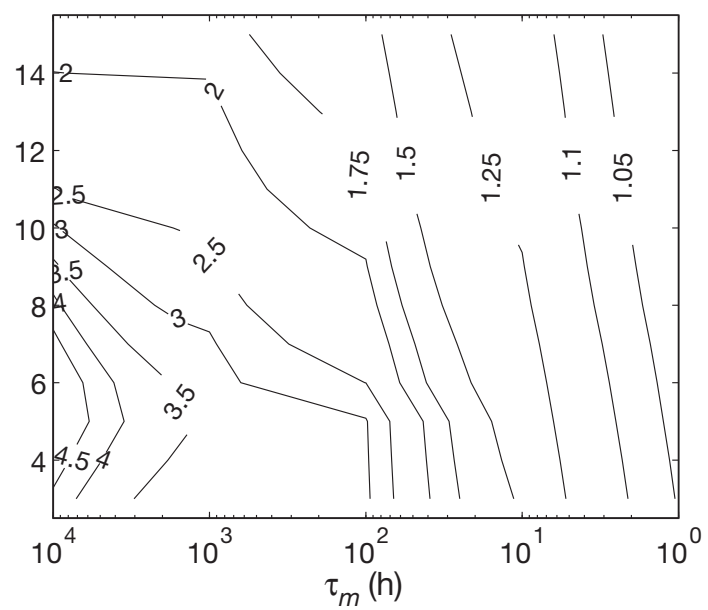

Fig. 6. Vertical heterogeneity $X_{\text {base }} / X_{\text {top }}$ between the top and base of a freely mixing SML for a range of mixing time scales, $\tau_{m}$, and optical mixing layer depths $\xi_{\text {SML }}$. The contours show the daily maximum vertical relative difference for (a) the Chl:C ratio and (b) the inhibition parameter $\vartheta$ (note that $\vartheta$ gives the portion of functional centres and is thus larger at depth)

cell division, is responsible for the slightly lower productivity values in Table 3.

To further elaborate on the differences between a mixed and a mixing layer, we can compare the vertical heterogeneity of some physiological indicators for a range of mixing time scales and optical depths. Fig. 6 shows that it becomes difficult to speak of a mixed layer already for mixing time scales of $\tau_{m} \geq 100 \mathrm{~h}$, despite the convective overturning at night. The vertical movement during the day is so small that the vertical heterogeneity in the physiology starts to exceed $50 \%$, i.e. the values for both Chl:C and $\vartheta$ can be more than $50 \%$ higher at the base of the SML compared to the surface (Figs. $4 d \& 6$ ). It should be noted that Fig. 6 shows the maximal difference that we observed over the course of a day. For the $\mathrm{Chl}$ : $\mathrm{C}$ ratio this typically occurs in the late afternoon, a few hours before sunset, while for the inhibition parameter $\vartheta$ the maximal difference is observed during the early afternoon (cf. Fig. 4). During other times of the day, the SML is more homogeneous than shown in Fig. 6.

\section{Conclusions}

(1) For SMLs that are optically shallow and only low to moderately turbulent, the depth-integrated productivity measurements from fixed-depth incubations provide a good estimate (error $<10 \%$ ) of the true in situ productivity (Figs. 1e \& 2), irrespective of the photophysiology of the cells (provided that a sufficiently large number of depths are sampled). Most coastal and oceanic SMLs should fall into this category (Region I in Fig. 2).
(2) For highly turbid SMLs, where the SML depth exceeds the euphotic depth by a factor 2 or more, the fixed-depth incubations can overestimate the depthintegrated in situ productivity by up to $25 \%$ (Figs. $1 \mathrm{~g}$ $\& 3 a)$. This value is highly variable, however, and depends on the environmental conditions, the photophysiology of the cells, and the incubation time, in particular:

(a) an increase in the photodamage probability $\left(k_{d}\right)$ in combination with a higher irradiance maximum leads to a reduction in the overall productivity but more so in the near-surface fixed-depth incubations (Fig. 3c). The effect is more pronounced in the stationary incubations as the freely mixing cells only experience the supra-optimal (and thus damaging) near-surface light intensities for a short period and are thus less inhibited. The result is a reversal in the depth-integrated error which changes from a $16.5 \%$ over- to a $14 \%$ underestimation (Fig. 3a-d).

(b) an increase in turbulence has a seemingly paradoxical effect: in the scenario of Fig. $1 \mathrm{~g}$ the error $\bar{\Pi}$ is essentially the same or even slightly larger than in Fig. 3a, although the mixing time scale in the latter scenario is 100 times lower. This seems paradoxical, since we would expect a higher turbulence intensity to always result in higher errors, as it amplifies the contrast between the fixed-depth incubations and the highly dynamic conditions in a freely mixing water column. The issue is more complex, however, as an increase in mixing intensity has several effects which may not be immediately apparent. Higher turbulence leads to (i) an increase in $P_{\text {mixed }}$ near the surface because higher mixing intensities lead to shorter residence times near the surface and thus 
lower levels of inhibition. This leads to a negative $\Pi$ at the surface, and a reduction of $\bar{\Pi}$. But we have an additional factor which counteracts the reduction in $\bar{\Pi}$, namely (ii) $P_{\text {mixed }}$ turning negative below the $1 \%$ light depth $(\zeta \geq 4.6)$ while $P_{\text {incub }}$ simply stays at zero (Fig. 3a-f). The reason for this discrepancy is that the cells which are rapidly mixed through the SML accumulate carbon reserves (see the carbon allocation model in Ross \& Geider 2009) while they are in the well-lit euphotic part of the SML, and when turbulence mixes them to greater optical depths, they draw on these reserves for maintenance, thereby reducing the amount of carbon per cell; this results in negative productivity values. When the turbulence is low, the cells are moved more slowly to these depths, which means that they already use up most of their reserves on the way down, so that $P_{\text {mixed }}$ is only slightly negative at greater depths, since the cells there are already at their subsistence threshold (Fig. 1g).

(a) Data from Fig.1b in Marra (1978)

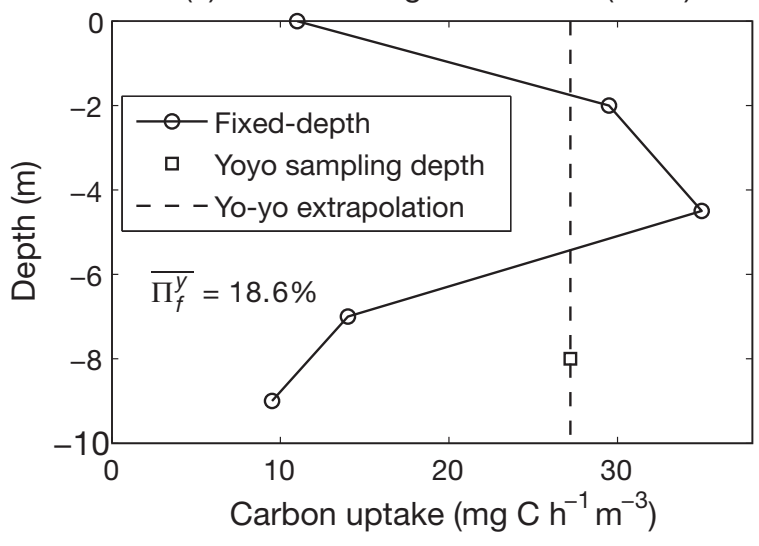

(c) Model simulation

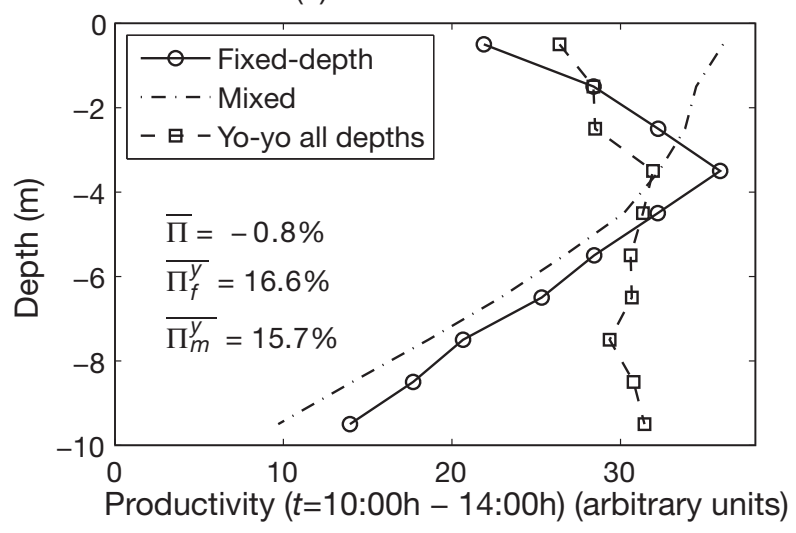

(c) a shorter incubation time typically leads to a reduction in the depth-integrated error (Fig. 5). However, short incubation times are difficult to interpret in terms of the daily production, as the extrapolation is not trivial. Shortening the incubation time is advisable only if the water column in question is likely to produce large errors (Fig. 2). When the error is expected to be low, a reduced incubation time has no effect on the already low errors (compare $\bar{\Pi}$ in Fig. 7c,d).

(3) If the turbulence is low to moderate, an increase $\xi_{\mathrm{SML}}$ leads to an increase in the depth-integrated error (compare Fig. 1h vs. b), which is mainly due to the fact that the overall productivity is reduced while the absolute error remains fairly constant in the euphotic zone, and thus the relative error $\Pi$ increases.

(4) While a reduction in the photosynthetic efficiency $\alpha_{\max }^{C h l}$ (Fig. 3e,f) does not affect the main shape of $\Pi$ (i.e. the signs of $\Pi$ at various depths remains almost unaffected), the depth-integrated error has been greatly

(b) Model simulation

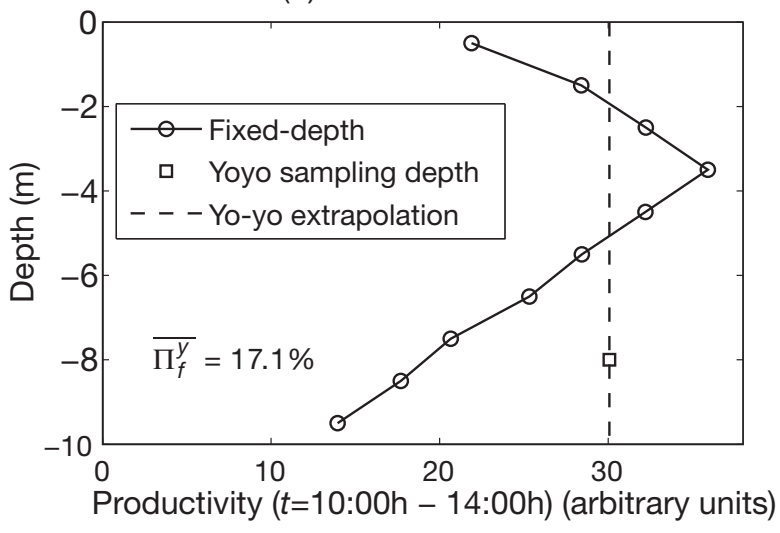

(d) Model simulation (24h incubation)

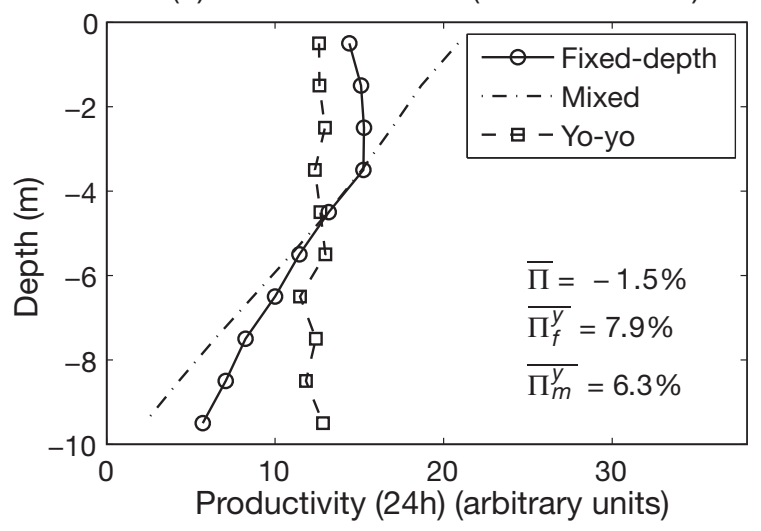

Fig. 7. (a) Observed productivity from Fig. $1 \mathrm{~b}$ in Marra (1978). (b) Simulation results for a 'similar' water column. $\bar{\Pi}_{f}^{y}$ gives the percent overestimation of the productivity between the yo-yo and fixed-depth incubations. (c) As in (b) but showing also the productivity in the freely mixing water column and the yo-yo productivity for water samples obtained at different depths. $\bar{\Pi}$ is the error defined in Eq. (9) while $\bar{\Pi}_{m}^{y}$ gives the percent error between the yo-yo incubations and the freely mixing water column. (d) As in (c) but using a full $24 \mathrm{~h}$ for the incubation 
reduced (compare Fig. 3f vs. b). This is mainly a mathematical effect: while the absolute error at the surface remains fairly constant, the productivity has dropped considerably and thus the same absolute error delivers a much larger relative error. Although $\Pi<0$ only in the surface bin, this is sufficient to reduce the overall error and even turn it negative.

These results, and in particular (1) above, contradict previous studies, which found a 'significant' under(Marra 1978, Gallegos \& Platt 1982, Gocke \& Lenz 2004) or overestimation (Barkmann \& Woods 1996) of in situ productivity by fixed-depth incubations. While our results tend to qualitatively agree with the overestimation ( $\bar{\Pi}$ was typically positive for low to moderate levels of photoinhibition and only turned negative for high damage probabilities $k_{d}$ ), we did not observe the $40 \%$ overestimation obtained with the modelling approach in Barkmann \& Woods (1996). The discrepancy might be related to differences in the treatment of photoinhibition. While our in vitro cells show significant signs of inhibition near the surface, due to a constant damage probability $k_{d}$, the amount of photoinhibition in the Barkmann \& Woods (1996) model depends on the acclimation state and is very low if the cells are fully adapted to high light. They state: 'Photoinhibition can occur close to the surface in the mixed-water column, but is negligible in the static one, since the lightadapted cells have a small $\beta$.' ( $\beta$ being their inhibition parameter). In our case the amount of inhibition was much higher in the static incubations compared to the freely mixing cells, as the mobile cells spend much less time at the high light intensities. We would obtain values for $\bar{\Pi}$ that are of the same order of magnitude $(40 \%)$ as in Barkmann \& Woods (1996), if we reduced our damage probability $k_{d}$ (not shown).

\section{Evaluation of the accuracy of yo-yo incubations}

Marra (1978) found that oscillating bottle incubations yielded productivity values that were up to $87 \%$ higher, compared to fixed-depth incubations. Fig. 1 of Marra (1978) shows the results from his 17 Sep experiment that showed the smallest discrepancy between the oscillating and fixed-depth incubations of $18.6 \%$. In order to be able to compare our results with Marra's vertical profile, we chose this experiment for a model simulation. The mixing layer was reported to be $10 \mathrm{~m}$, and irradiance at the bottom of the mixing layer was reported to be $5 \%$ of the surface value. From this, we calculated an attenuation coefficient of $k=0.3 \mathrm{~m}^{-1}$. The resulting mixing layer is thus located in Region I in Fig. 2 and we would expect only very small errors $(<10 \%)$. Marra (1978) filled the bottles for fixed-depth incubations from samples taken at $0,2,4.5,7$ and $9 \mathrm{~m}$ whereas the bottle for cycling through the mixing layer was collected from a depth of $8 \mathrm{~m}$. Based on the sampling protocol in Marra (1978) we chose a vertical velocity of $5.4 \mathrm{~m} \mathrm{~h}^{-1}$ for the oscillating bottle. In our simulation, we used the parameters for Skeletonema costatum (Table 1), except that we increased $k_{d}$ to $3 \times$ $10^{-7}$ in order to increase the amount of photoinhibition at the surface. The result of this simulation (Fig. 7b) shows good qualitative agreement with Marra's experimental results (Fig. 7a) in that the extrapolated productivity in the oscillating bottle yields a productivity estimate that is higher than the depth integrated productivity from the fixed-depth incubations. The discrepancy between the oscillating and fixed-depth productivity can be further increased if we increase the surface irradiance. In Fig. $7 \mathrm{~b}$ we used a noon maximum of $650 \mathrm{~W} \mathrm{~m}^{-2}$ which resulted in about $1200 \mu \mathrm{mol}$ photons $\mathrm{m}^{-2} \mathrm{~s}^{-1}$ of PAR. If we increase this value to $850 \mathrm{~W} \mathrm{~m}^{-2}$ (equivalent to about $1600 \mu \mathrm{mol}$ photons $\mathrm{m}^{-2}$ $\mathrm{s}^{-1}$ of PAR) the discrepancy increases from $17.1 \%$ to over $30 \%$. We also tried higher and lower values for $K_{m}$ but found that our error did not change significantly. We cannot be confident that we have simulated the 'true' conditions in the experiments conducted by Marra (1978) because we lack information on the composition of the phytoplankton community, its physiology and the in situ turbulence and light environment.

While observational oceanographers are typically limited by logistic constraints, such as the number of samples that can be taken (and processed) in a certain period of time, the modeller is only limited by the simplifying assumptions that went into the construction of the model. Thus while Marra (1978) used one oscillating incubation bottle with a sample population collected from a particular depth, we can simulate a series of oscillating bottles with sample populations that originated from different depths as well as the in situ productivity in the freely mixing water column (Fig. 7c). For the oscillating bottles we notice that the choice of the sampling depth has an effect on the productivity estimate (dashed line with open squares in Fig. 7c). If we were to take the sample population from the surface, for instance, and also start the oscillation there, the productivity in this oscillating bottle would be about $13 \%$ lower compared to the one that started at $8 \mathrm{~m}$ depth. A possible explanation for this discrepancy is that samples near the surface have a lower Chl:C ratio and therefore show a reduced (short term) productivity if exposed to the same light intensity as samples taken from greater depths that possess more chlorophyll.

Nevertheless, the question we should really be asking is: does the yo-yo approach approximate the depthintegrated in situ productivity estimates better than fixed-depth incubations? For the results we presented 
here, and in particular for the water column in Fig. 7, the answer is no. If we compare the freely-mixed with the fixed-depth productivity (Fig. 7c) the error is negligible $(\bar{\Pi}=-0.8 \%)$. Thus by using the yo-yo approach we have actually increased the error from $0.8 \%$ to $17.1 \%$, and even if we would use more than one oscillating bottle, the error would still be larger than from fixed-depth incubations. The same is true if we increase the sampling period from the $4 \mathrm{~h}$ in Marra (1978) to a full $24 \mathrm{~h}$ (Fig. 7d). The effect of photoinhibition is that the fixed-depth productivity is lower near the surface and higher at depth. The latter is due to the fact that in the fixed-depth incubations the cells tend to have a lower inhibition level at depth than their freely mixing counterparts (compare the mixed and stationary examples in Fig. 4b). The vertical heterogeneity among different oscillating bottles as well as the discrepancy between the oscillating bottles and the fixed or mixed productivity can be considerably reduced if we choose a longer incubation period (Fig. 7d).

In Ross et al. (2011), we found considerable errors (of the order of $100 \%$ ) in growth rate estimates from fixeddepth incubations, whereas in the present study our errors are considerably smaller. Measurements of growth rate from $\mathrm{Chl}$ increase in bottle incubation experiments are typically made at only one light level (one depth) and thus care needs to be taken in choosing the depth to ensure that results are representative of the entire mixing layer. Failure to choose the correct depth could produce the large errors reported in Ross et al. (2011). In contrast, estimates of primary production are usually based on ${ }^{14} \mathrm{C}$ in a series of samples that are incubated at fixed depths throughout the mixing layer. Here, the concern is not in choosing the correct light level, but in whether stranding samples at fixed depth biases the measured $\mathrm{CO}_{2}$ fixation relative to the in situ mixed conditions. As we could show, the effect of arresting the cells at fixed depths only introduces a small systematic error to the results. We would find equally small errors in the Chl-based growth rate experiments if we were to use more than one sampling depth. The growth rates for the entire water column ( $\bar{\mu}$ in Ross et al. 2011) generally showed a good agreement both between the fixed-depth and freely mixing scenarios, but also between the Chl- and carbonderived equivalents (Ross et al. 2011).

Acknowledgements. The research leading to these results received funding from the European Union Seventh Framework Programme (FP7/2007-2013) Marie Curie Intra-European Fellowship for Career Development under grant agreement no. 255396 for project AQUALIGHT (www.aqualight. info). R.J.G.'s research on photoacclimation is supported by the UK Natural Environment Research Council (NE/ G003688/1). The authors also acknowledge the Spanish funded SUMMER (CTM200803309/MAR) and ANERIS (PIF08-015) projects.

\section{LITERATURE CITED}

Barber R, Hilting A 2002. History of the study of plankton productivity. In: Willams P, Thomas D, Reynolds C (eds) Phytoplankton productivity: carbon assimilation in marine and freshwater ecosystems. Blackwell, Oxford, p 16-43

Barkmann W, Woods JD (1996) On using a Lagrangian model to calibrate primary production determined from in vitro incubation measurements. J Plankton Res 18:767-788

Behrenfeld MJ, Prasil O, Kolber ZS, Babin M, Falkowski PG (1998) Compensatory changes in photosystem II electron turnover rates protect photosynthesis from photoinhibition. Photosynth Res 58:259-268

Eilers PHC, Peeters JCH (1988) A model for the relationship between light intensity and the rate of photosynthesis in phytoplankton. Ecol Model 42:199-215

Falkowski PG, Barber RT, Smetacek V (1998) Biogeochemical controls and feedbacks on ocean primary production. Science 281:200-206

Friend AD, Geider RJ, Behrenfeld MJ, Still CJ (2009) Photosynthesis in global-scale models. In: Laisk A, Nedbal L, Govindjee (eds) Towards photosynthesis in silico. Springer, Dordrecht, p 465-497

Furnas M (2002) Measuring the growth rates of phytoplankton in natural populations. In: Rao DVS, Rao S, Ganapati PN, Lafond EC, Humphrey GF (eds) Pelagic ecology methodology. CRC Press, Boca Raton, FL, p 221-249

Gallegos CL, Platt T (1982) Phytoplankton production and water motion in surface mixed layers. Deep-Sea Res I 29: 65-76

Gallegos CL, Platt T (1985) Vertical advection of phytoplankton and productivity estimates: a dimensional analysis. Mar Ecol Prog Ser 26:125-134

Geider RJ, MacIntyre HL, Kana TM (1998) A dynamic regulatory model of phytoplanktonic acclimation to light, nutrients, and temperature. Limnol Oceanogr 43:679-694

Gocke K, Lenz J (2004) A new turbulence incubator for measuring primary production in non-stratified waters. J Plankton Res 26:357-369

Harris GP, Smith REH (1977) Observations of small-scale spatial patterns in phytoplankton populations. Limnol Oceanogr 22:887-899

Hendricks MB, Bender ML, Barnett BA, Strutton P, Chavez FP (2005) Triple oxygen isotope composition of dissolved $\mathrm{O}_{2}$ in the equatorial Pacific: a tracer of mixing, production, and respiration. J Geophys Res 110:C12021. doi:10.1029/ 2004JC002735

Köhler J (1997) Measurement of in situ growth rates of phytoplankton under conditions of simulated turbulence. J Plankton Res 19:849-862

Köhler J, Schmitt M, Krumbeck H, Kapfer M, Litchman E, Neale PJ (2001) Effects of UV on carbon assimilation of phytoplankton in a mixed water column. Aquat Sci 63: 294-304

> Lewis MR, Cullen JJ, Platt T (1984a) Relationships between vertical mixing and photoadaptation of phytoplankton: similarity criteria. Mar Ecol Prog Ser 15:141-149

Lewis MR, Horne EPW, Cullen JJ, Oakey NS, Platt T (1984b) Turbulent motions may control phytoplankton photosynthesis in the upper ocean. Nature 311:49-50

Li WKK, Maestrini SY (1993) Measurement of primary production from the molecular to the global scale. ICES Symposium 197. ICES Secretariat, Copenhagen

> Lohrenz SE, Weisenburg DA, Rein CR, Arone RA, Taylor CT, Knauer GA, Knap AH (1992) A comparison of in situ and simulated in situ methods for estimating oceanic primary production. J Plankton Res 14:201-221 
Long SP, Humphries S, Falkowski PG (1994) Photoinhibition of photosynthesis in nature. Annu Rev Plant Physiol Plant Mol Biol 45:633-662

MacIntyre HL, Kana TM, Geider RJ (2000) The effect of water motion on short-term rates of photosynthesis by marine phytoplankton. Trends Plant Sci 5:12-17

Mallin M, Paerl H (1992) Effects of variable irradiance on phytoplankton productivity in shallow estuaries. Limnol Oceanogr 37:54-62

Marra J (1978) Phytoplankton photosynthetic response to vertical movement in a mixed layer. Mar Biol 46:203-208

Moigis AG (2000) Photosynthetic rates in the surface waters of the Red Sea: the radiocarbon versus the non-isotopic dilution method. J Plankton Res 22:713-727

Parsons TR, Maita Y, Lalli CM (1985) A manual of chemical and biological methods for seawater analysis. Pergamon Press, Oxford

Pemberton KL, Clarke KR, Joint I (2006) Quantifying uncertainties associated with the measurement of primary production. Mar Ecol Prog Ser 322:51-59

Richardson K, Samuelsson G, Hällgren JE (1984) The relationship between photosynthesis measured by ${ }^{14} \mathrm{C}$ incorporation and by uptake of inorganic carbon in unicellular algae. J Exp Mar Biol Ecol 81:241-250

Editorial responsibility: Katherine Richardson, Copenhagen, Denmark
Ross ON, Geider RJ (2009) New cell-based model of photosynthesis and photo-acclimation: accumulation and mobilisation of energy reserves in phytoplankton. Mar Ecol Prog Ser 383:53-71

Ross ON, Moore CM, Sugget DJ, MacIntyre HL, Geider RJ (2008) A model of photosynthesis and photo-protection based on reaction centre damage and repair. Limnol Oceanogr 53:1835-1852

Ross ON, Geider RJ, Berdalet E, Artigas ML, Piera J (2011) Modelling the effect of vertical mixing on bottle incubations for determining in situ phytoplankton dynamics. I. Growth rates. Mar Ecol Prog Ser 435:13-31

Steemann-Nielsen E (1952) The use of radioactive carbon $\left(\mathrm{C}^{14}\right)$ for measuring organic carbon production in the sea. J Cons Int Explor Mer 18:117-140

Strickland JDH, Parsons TR (1977) A practical handbook of seawater analysis. Vol 167. Fisheries Research Board of Canada, Ottawa

Tokarev YN, Williams R, Piontkovski SA (1998) Small-scale plankton patchiness in the Black Sea euphotic layer. Hydrobiologia 375/376:363-367

> Yoder JA, Bishop SS (1985) Effects of mixing-induced irradiance fluctuations on photosynthesis of natural assemblages of coastal phytoplankton. Mar Biol 90:87-93

Submitted: September 30, 2010; Accepted: May 2, 2011

Proofs received from author(s): August 12, 2011 